\title{
Small variation of annual temperature in deep tunnel can produce annual variation in tilt and strain*
}

\author{
SUN Yu-jun ${ }^{1), *}$ (孙玉军) LI Jie $^{2)}$ (李 杰) CAO Jian ling ${ }^{1)}$ (曹建玲) \\ LU Shuang-ling ${ }^{3)}$ (卢双苓) SHI Yao lin ${ }^{1)}$ (石耀霖) \\ 1) Laboratory of Computational Geodynamics, Graduate University of the Chinese Academy of Sciences, Beijing \\ 100049, China \\ 2) Earthquake Administration of Shandong Province, Ji' nan 250014, China \\ 3) The Standard Seismic Station of Taian, Taian 271600, China
}

\begin{abstract}
In this paper, finite element method (FEM) of axisymmetric linear elastic model has been used to calculate the tilt and strain induced by small annual temperature variations in a deep tunnel. The results show that even if the amplitude of the annual variation meets the construction standard of seismic station issued by China Earthquake Administration (the annual temperature variation amplitude in the tunnel is no more than $0.5^{\circ} \mathrm{C}$ ), a small annual temperature variation of amplitude just $0.2{ }^{\circ} \mathrm{C}$ in the tunnel would produce $10^{-7} \mathrm{rad}$ changes in tilt and $10^{-7}$ changes in strain. Especially, at the end and the corner of the tunnel, changes of tilt and strain can be even larger. Therefore, in the future, it is an important task to reduce the annual temperature variation in the tunnel as far as possible. Within the tunnel, for both baseline instrument and pendulum instrument, the modeling suggests ways of construction of the tunnel and installation of the instrument to decrease the influence of the annual temperature variation.
\end{abstract}

Key words: finite element method (FEM); tilt; strain; annual variation

CLC number: $\mathrm{P} 315.72^{+} 7 \quad$ Document code: A

\section{Introduction}

Tilt and strain observations in seismic crustal deformation stations are the main source of data to analyze earthquake precursor anomaly. The observation conditions in the tunnel are relatively good. The effects of temperature, humidity, atmosphere pressure, gale, rainfall and other meteorological factors on the observation results are small, and surface noise is significantly reduced in the tunnel. Therefore, instruments installed in tunnels can carry out continuous automatic observation and the accuracy is relatively high. Observations in tunnels play an important role in earthquake monitoring. However, in the tunnel where observation conditions are good, what interference factors can still play a dominant role? And what degree of impact on the measurement results might be caused with these interference factors? Researches to better understand these problems has an im-

\footnotetext{
Received 2008-01-25; accepted in revised form 2008-07-21.

Foundation item: China Earthquake Administration Sector Fund (2008419077) and Knowledge Innovation Program of the Chinese Academy of Sciences (kzcx2-yw-123).

* Author for correspondence: sunyujunabc@163.com
} 
portant role to eliminate the interference factors, highlight the anomaly response for earthquake, determine appropriate observation conditions and instrument precision selection.

In order to identify anomalies, it is essential to understand various factors which may influence the deformation measurement, including climate change, human activities, equipment faults and many unknown cases. With the current cognitive power, it's difficult to distinguish the difference between interference from the changes with what are regarded as earthquake precursors (WANG et $a l$, 2004a). Among these interference factors, climate changes mainly include atmospheric pressure, temperature, rainfall, gale and thunder. Human activities include load and surface noise. Equipment faults are mainly about the instrument drift. In regard to the quantitative research on various interference factors, scholars worldwide have done a lot of work. With observation data of volume strain gauge in Huailai crustal deformation station, WANG et al (2004b) analyzed the effect of atmospheric pressure and drift and provided a number of methods for eliminating interference and data processing methods. MOU (1999) gave some data processing methods about the instrument drift with the observation results of volume strain gauge in the Xichang seismic station. HU et al (2002) did some experiments about the effect of load in the surface on the strain and stress. Using the observation data in the area of Friluli, Italy, Zadro et al (1988), Moro and Zadro (1998), Garavaglia et al (2000) did some research about how atmospheric pressure, rainfall and temperature impact on the strain and stress. Furthermore, seismic stations have different response to various interference factors. Generally, to a station, it usually responds dominantly to one or two interference factors. WANG and JI (2000) have analyzed the anomaly characters at many seismic stations in Shangdong Province and gave some conclusions. For example, the Yantai station is obviously influenced by the atmospheric pressure and rainfall while the Taian station is affected by the temperature obviously.

Among these interference factors, the impact of temperature is undoubtedly an important factor. Previous works have noticed the changes of tilt and strain due to temperature variation. However, they are mostly limited to analyze the relation of them, but did not give a quantitative analysis on the physical mechanism. In recent years, some researchers begin to focus on this quantitative research about the physical mechanism. CAO and SHI (2005) have done some quantitative research about the stress and tilt due to the surface annual temperature variation, in the case of topography, with finite element method. Furthermore, SUN et al (2008) have calculated and analyzed the strain and tilt due to surface annual temperature variation using the observation data from Taian seismic station.

To the deformation measurement in the tunnel, the China Earthquake Administration (2004) has issued the construction standard of seismic station; there are some rules to guarantee the observation condition in the tunnel and instrument installation. The annual temperature variation amplitude in the tunnel is required no more than $0.5^{\circ} \mathrm{C}$ and the amplitude of daily variation is required no more than $0.03^{\circ} \mathrm{C}$. But can we eliminate the influence of temperature change if we meet the requirement of specification? In this paper, we apply finite element method (FEM) to calculate the strain and tilt due to the small annual temperature variation in the tunnel which meets the official standard requirement, and discuss how to reduce the impact of annual temperature variation based on our calculation results.

\section{Model and methodology}

\subsection{Calculation model}

Consider a semi-infinite half-space in the region $y \geq 0$. The surface is defined by the plane $y=0$. If we assume that the surface temperature $T$ is a periodic function of $\omega, T=T_{0}+\Delta T \sin (\omega t)$. Then the subsurface temperature is (Turcotte and Schubert, 1986) 


$$
T=T_{0}+\Delta T \exp \left(-y \sqrt{\frac{\omega}{2 \kappa}}\right) \sin \left(\omega t-y \sqrt{\frac{\omega}{2 \kappa}}\right)
$$

Where $T_{0}$ is the surface average temperature, $\Delta T$ is amplitude of surface temperature, $\kappa$ is thermal diffusivity, $t$ is time, $y$ is depth. With this equation, given $\omega=2 \times 10^{-7} \mathrm{rad} \cdot \mathrm{s}^{-1}, \kappa=1 \times 10^{-6} \mathrm{~m}^{2} \cdot \mathrm{s}^{-1}$, when amplitude of surface temperature $\Delta T=10^{\circ} \mathrm{C}$ and $y=50 \mathrm{~m}$, the subsurface temperature is only $1.37 \times 10^{-6}{ }^{\circ} \mathrm{C}$. Therefore, if the depth of the tunnel is deep enough, the influence of surface temperature annual variation can be neglected. Therefore, in the following model we will no longer discuss the effect of the surface temperature and we mainly study the question of tilt and strain due to small annual temperature variation in the tunnel.

As the structure of actual tunnels is very complicated, we have to generate a large number of grids in the 3-D FEM simulation if we want to get a relative high accuracy results. As a result the computational complexity is too large to carry out with serial computer code. Therefore, in this paper we make some appropriate assumption and give a relatively simple model. We consider a simple case: First excavate a shaft, and then dig some horizontal tunnels. In this model, neglecting

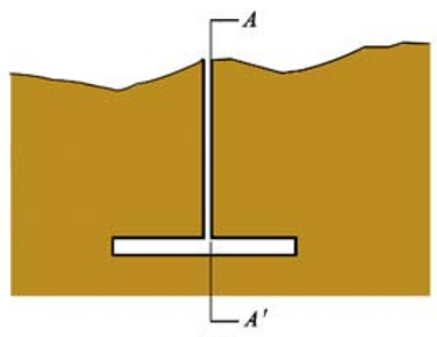

(a)

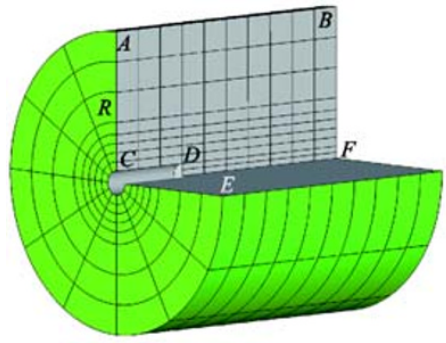

(c)

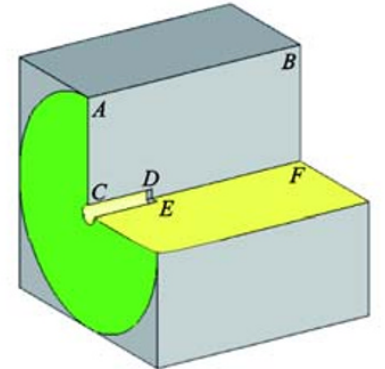

(b)

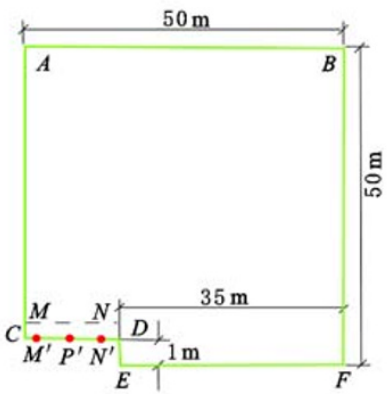

(d)

Figure 1 Model establishment

(a) Schematic diagram of the tunnel; (b) Simplification of the axisymmetric issue; (c) Schematic diagram of the mesh; (d) Calculation model and its sizes

the influence of the shaft, we just consider the effect of temperature in the horizontal tunnels. And the left and right tunnels are symmetrical to the shaft. So we just consider the right part of the shaft axis $A A^{\prime}$ (see Figure 1a). Assuming that the horizontal tunnel has a diameter of $2 \mathrm{~m}$ and the length of it is $15 \mathrm{~m}$. Since the 3-D structure of actual tunnel is complicated, further study on the right of the model is to regard it as an axisymmetric problem (see Figure 1b). That is we consider the axis of the horizontal tunnel as the symmetrical axis of the model. The plane of $B A C D E F$ ro- 
tates $360^{\circ}$ around the axis of $E F$ to get a spatial cylinder (To see it clearly, we give a three-quarter of the cylinder). If $C A$ is large enough, we can neglect the effect of the outer cylinder temperature and the surface temperature (Considering the linear problem can be superimposed, we can consider it separately). To the 3-D axisymmetric problem, the deformation of the plane represents the entire deformation of the cylinder. So we finally simplify the model as a 2-D plane (see Figure 1d). The horizontal length of the model is $50 \mathrm{~m}$. The radius of the cylinder is also $50 \mathrm{~m}$. EF is the symmetrical axis. Temperature boundary conditions of the model are given as: temperature of $C D$ and $E F$ which are the wall of the tunnel change as $T=0.2 \sin (\omega t)$, other boundary are adiabatic. Mechanical boundary conditions of the model are given as the horizontal displacement of $A C$ and $B F$ is 0 and the vertical displacement is free. $C D, E F$ and $A B$ are all free boundaries.

\subsection{Equations}

In the cylindrical coordinate system $r, \theta, z$ ( $z$ is the symmetrical axis), variables in axisymmetric problem have no relation with $\theta$. Shear stress $\tau_{r \theta}, \tau_{z \theta}$ and displacement $u_{\theta}$ are all equal to 0 . Therefore, the equations can be simplified (LU and LUO, 1990). We consider the medium as linear elastic and calculate them with following equations:

Heat transfer equation

$$
\frac{\partial T}{\partial t}=\kappa\left\{\frac{1}{r} \frac{\partial}{\partial r}\left(r \frac{\partial T}{\partial r}\right)+\frac{\partial^{2} T}{\partial z^{2}}\right\}
$$

Equilibrium equations (no body force)

$$
\frac{\partial \sigma_{\gamma}}{\partial r}+\frac{\partial \tau_{\gamma z}}{\partial z}+\frac{\partial_{\gamma}-\partial_{\theta}}{r}=0, \frac{\partial \tau_{z \gamma}}{\partial r}+\frac{\partial \sigma_{z}}{\partial z}+\frac{\tau_{z \gamma}}{r}=0
$$

Compatibility equations

$$
\varepsilon_{r}=\frac{\partial u_{\gamma}}{\partial r}, \quad \varepsilon_{\theta} \frac{u_{\gamma}}{r}, \quad \varepsilon_{z}=\frac{\partial w}{\partial z}, \quad \gamma_{z \gamma}=\frac{\partial w}{\partial r}+\frac{\partial u_{\gamma}}{\partial z}
$$

Constitutive equations

$$
\left\{\begin{array}{l}
\sigma_{\gamma} \\
\sigma_{\theta} \\
\sigma_{z} \\
\tau_{\gamma z}
\end{array}\right\}=\frac{E}{(1+\mu)(1-2 \mu)}\left[\begin{array}{cccc}
1-\mu & \mu & \mu & 0 \\
\mu & 1-\mu & \mu & 0 \\
\mu & \mu & 1-\mu & 0 \\
0 & 0 & 0 & 0.5-\mu
\end{array}\right]\left\{\begin{array}{l}
\varepsilon_{\gamma} \\
\varepsilon_{\theta} \\
\varepsilon_{z} \\
\gamma_{\gamma z}
\end{array}\right\}-\frac{E \alpha_{1} T}{1-2 \mu}\left\{\begin{array}{l}
1 \\
1 \\
1 \\
0
\end{array}\right\}
$$

The temperature condition in the horizontal tunnel is

$$
T=0.2 \sin (\omega t)
$$

Where $T$ is temperature; $t$ is time; $\kappa$ is thermal diffusivity; $u_{r}$ and $w$ are the displacement at the direction of $r$ and $z ; \varepsilon_{r}, \varepsilon_{\theta}, \mathcal{E}_{z}$,are the normal strain at the direction of $r, \theta, z ; \gamma_{z r}$ is shear stress; $\sigma_{r}$, $\sigma_{\theta}, \sigma_{z}$ are the normal stress at the direction of $r, \theta, z ; \tau_{z r}$ is shear stress, $E$ is elastic modulus, $\mu$ is Poisson's ratio, $\alpha_{1}$ is thermal expansion coefficient. We use international system of units and $\kappa=1 \times 10^{-6} \mathrm{~m}^{2} \cdot \mathrm{s}^{-1}, \alpha_{1}=10^{-5} \mathrm{~K}^{-1}, E=70 \mathrm{GPa}, \mu=0.25, \omega=2 \times 10^{-7} \mathrm{rad} \cdot \mathrm{s}^{-1}$ in this calculation.

\section{Results and discussion}

Usually, in the crustal deformation stations, there are two kinds of instruments: One is a baseline instrument which is to measure tilt and strain by observing the change of two points' position. 
Between the two points is the baseline. The other is a pendulum instrument whose work principle is to measure the change of pendulum's angle to one point. According to these principles we analyze the results of our calculation. For the baseline instrument, we set the observe points below ground level a certain distance actually. So we select $M$ and $N$ as two observe points of the baseline. $M$ and $N$ are at the same horizontal level which has $0.2 \mathrm{~m}$ from the line of $C D$, the wall of horizontal tunnel (see Figure 1d). For the pendulum instrument, we select three points on the line of $C D$ : $M^{\prime}, P^{\prime}, N^{\prime}$. The horizontal distance between $M^{\prime}$ and $N^{\prime}$ is $12 \mathrm{~m}$. From $M^{\prime}$ to the point $C$ is $1 \mathrm{~m}$ and from $N^{\prime}$ to the end of the tunnel is $2 \mathrm{~m}$. P' is the horizontal center of line $M^{\prime} N^{\prime}$. For the baseline instrument, considering $M$ and $N$ as two observe points, we calculate tilt and strain with equations:

$$
\begin{aligned}
& \alpha_{L}=\frac{\Delta v_{M}-\Delta v_{N}}{L} \\
& \varepsilon_{L}=\frac{\Delta u_{M}-\Delta u_{N}}{L}
\end{aligned}
$$

Where $L$ is the distance between $M$ and $N . u$ is velocity of horizontal direction and $v$ is velocity of vertical direction.

For the pendulum instrument, taking $P^{\prime}$ as an example, we calculate tilt and strain with equations:

$$
\begin{gathered}
\alpha_{P^{\prime}}=\frac{\frac{\partial v_{P^{\prime}}}{\partial x}}{1+\frac{\partial u_{P^{\prime}}}{\partial x}} \\
\varepsilon_{P^{\prime}}=\frac{\partial u_{P^{\prime}}}{\partial x}
\end{gathered}
$$

Finite element method (FEM) is employed to calculate and analyze the model. We adopt the element type is quadrilateral and generate more grids near the tunnel. The element number is 149301 and the node number is 149500 . We calculate for 6 periods (equivalent 6 years) and the time step is 1 day. After the results departure from the effect of initial transient and close to stability cyclical changes, we take the final period for analysis.

We set the distance between $M$ and line $A C$ to be $1 \mathrm{~m}$ and change the position of $N$, making the distance between $M$ and $N$ respectively equal to $6 \mathrm{~m}, 9 \mathrm{~m}$, and $12 \mathrm{~m}$. Considering $M N$ as baseline, we calculate tilt and strain with equations (7) and (8) for the three different baseline lengths. The results are shown in Figure 2. Although perturbation of temperature in the tunnel is small, it produces the magnitude of tilt up to $10^{-7} \mathrm{rad}$ and strain up to $10^{-7}$. These changes can have effect on the actual data because the accuracy of instrument is $10^{-9}$ rad to tilt and $10^{-9}$ to strain (construction standard of seismic station in China, 2004). According to the results, when we meet the standard requirement: the amplitude of annual temperature variation is no more than $0.5^{\circ} \mathrm{C}$, the changes of tilt and strain due to the small perturbations of annual temperature is obvious, and this effect cannot be neglected.

Additionally, in Figure 2 it is shown that tilt and strain due to the annual temperature variation are different to various length of baseline. Therefore, an appropriate length of baseline and two end points can be selected to reduce the effect of the annual temperature variation. What 

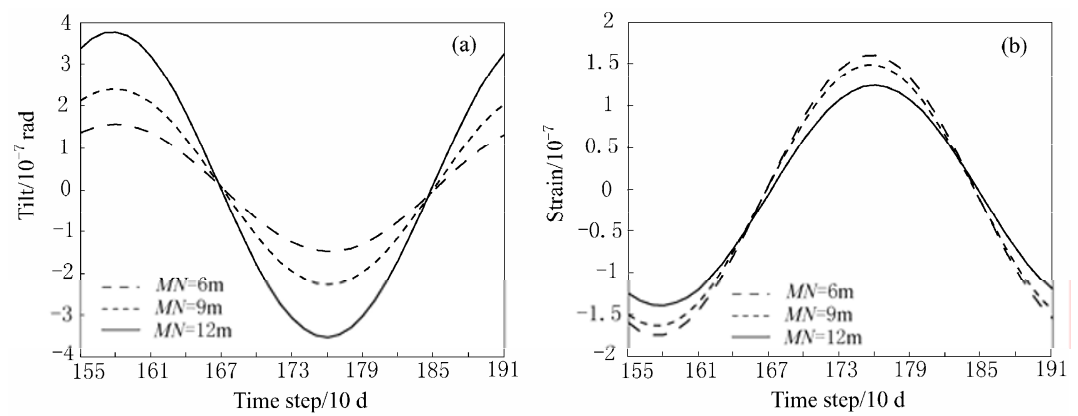

Figure 2 Results of tilt and strain vary with time steps based on the baseline $M N$

length of the baseline is reasonable? Figure 3 shows that tilt and strain which are calculated with equations (9) and (10) vary with the length of tunnel (The 0 point is entrance of the tunnel) at the last period to different steps: 1910 days, 1850 days, 1760 days, 1670 days and 1580 days. Figure 3 suggests that with the increase of the length of the tunnel, especially at the end of the tunnel (to tilt at the length of 11 14 $\mathrm{m}$ and to stain at the length of $14 \mathrm{~m}$ ), tilt and strain increase sharply. Tilt increase to $10^{-6} \mathrm{rad}$ from $10^{-8} \sim 10^{-7} \mathrm{rad}$ and strain increase to $10^{-6}$ from $10^{-8} \sim 10^{-7}$. The same phenomenon will happen at the corner of the tunnel. Figure 4 shows the contour of tilt and strain which are calculated by equations (9) and (10) at 1910 days. Because tilt and strain change very little far from the tunnel so we only select a region near to the tunnel. The selected region with computation mesh is shown in Figure 4. From Figures 4a and $4 \mathrm{~b}$ we can see that tilt and strain increase near to the tunnel. The distribution of temperature is uneven because of the tunnel. And the annual temperature variation will lead to heat convergence and diffusion. So this result in that tilt and strain are different at the same level. CAO and SHI (2005) pointed out that there are heat convergence and diffusion at the top or foot of the mountain. So the case at the corner or the end of the tunnel is similar. Heat convergence and diffusion will produce the changes of tilt, strain and stress. And this make there are fluctuation in our observation data. So to reduce the influence of annual temperature variation, we should avoid setting the two baseline points at the corner or the end of the tunnel. Especially for the baseline instrument, the space of the tunnel should be sufficient. The two baseline points should be installed at the center of the horizontal tunnel as far as possible to avoid the effect of annual temperature variation. To the requirement of the construction standard of seismic station in China (2004), the length of the baseline instrument room should be no less than $10 \mathrm{~m}$, width $2 \mathrm{~m}$ and height $2.5 \mathrm{~m}$; the length of the pendulum instrument room should be no less than $3 \mathrm{~m}$, width $2 \mathrm{~m}$ and height $2.5 \mathrm{~m}$. Additionally, considering symmetry of the model (see Figure 1b), we can set two baseline points symmetrically to the axis of the shaft (see Figure 1a) because the results shown in Figure 3 also suit to the right region of the axis of shaft if the actual tunnel is the same with our model. So to these two baseline points, tilt and strain due to annual temperature variation in the tunnel is the same and changes will be eliminated when calculate with equations (7) and (8). So for the baseline instrument, we can reduce the influence of annual temperature variation in the tunnel by selecting two baseline points appropriately.

According to pendulum instrument, Figure 5 shows that tilt and strain which are calculated with equations (9) and (10) change with time steps at three points: $M^{\prime}, P^{\prime}$ and $N^{\prime}$. As discussed above, heat convergence and diffusion have greater impact on the pendulum instrument. Tilt of point $M^{\prime}$ which is at the entrance of the tunnel is $10^{-8} \mathrm{rad}$ which increased to $10^{-6} \mathrm{rad}$ sharply at the 

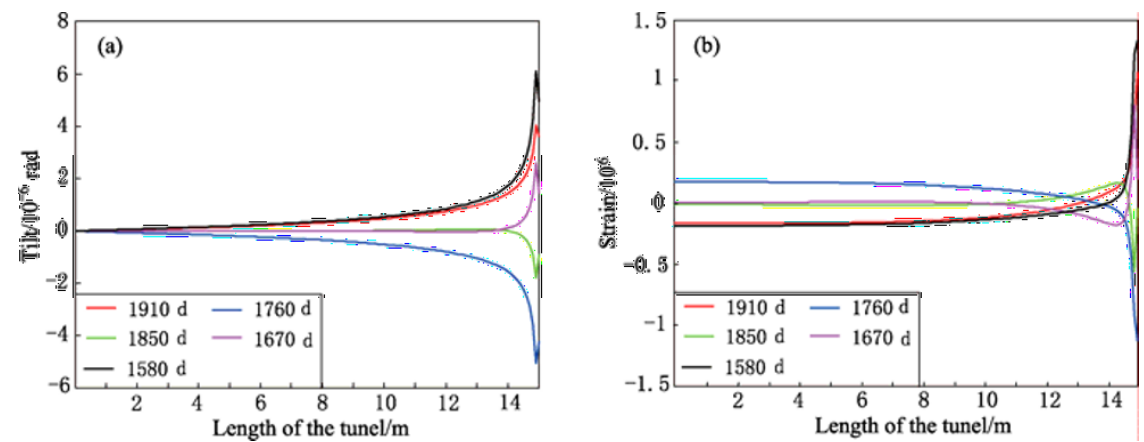

Figure 3 Tilt and strain vary with the length of the tunnel at different time steps

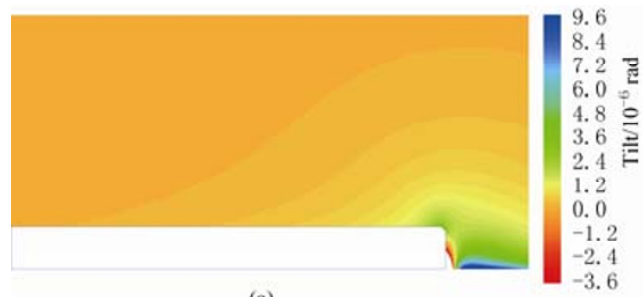

(a)

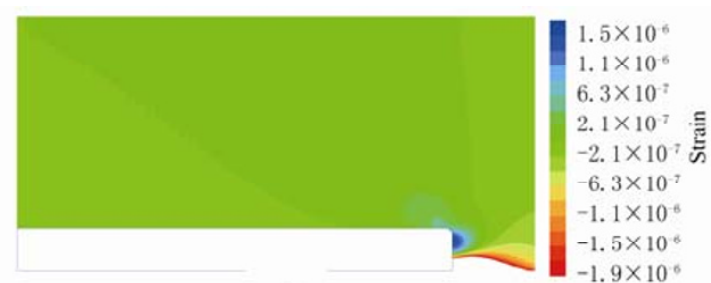

(b)

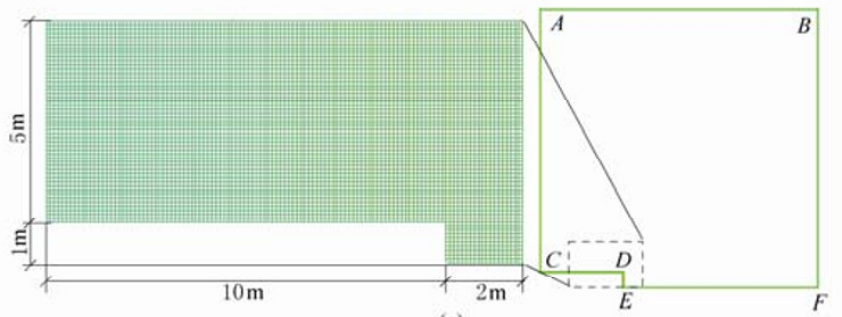

(c)

Figure 4 The contour of tilt and strain at 1910 days and part mesh of the model (a) The contour of tilt; (b) The contour of strain; (c) Part mesh of the model

points $N^{\prime}$ which is at the end of the tunnel (Figure 5a). So for the pendulum instrument, the position of the instrument installation is very important. Actually, we can select the position to install instrument where annual temperature variation has little influence on tilt and strain and avoid the corner or the end of the tunnel where heat convergence and diffusion is most obvious. The accuracy of the pendulum instrument is always very high but it is easy to be affected by surface factors. Some Japanese researchers thought it is not instability because it only reflects the measuring point (ZENG, 1984). In recent years, some high-precision, intelligent and network instrument have been produced and the accuracy of observation and resistance to jamming are also greatly enhanced (WANG et al, 2005, XIAO et al, 2005, SHI and WU, 2007). Strain due to annual temperature variation in the tunnel is very little if we select a reasonable point (Figure 5b). In fact, volume strain gauge can measure the strain to a point.

Comparing the calculation results of baseline (Figure 2) and pendulum (Figure 5), we find that the annual changes to these two instruments are different. The analysis of tilt indicate the magnitude of annual changes is $10^{-7} \mathrm{rad}$ in Figure 2a, but the magnitude ranges $10^{-8} \sim 10^{-6} \mathrm{rad}$ in Figure 5a. The analysis of strain indicate the magnitude of annual changes is $10^{-7}$ in Figure $2 \mathrm{~b}$, but the magnitude ranges $10^{-6} \sim 10^{-7}$ in Figure $5 \mathrm{~b}$. Therefore, from the same instrument at various loca- 

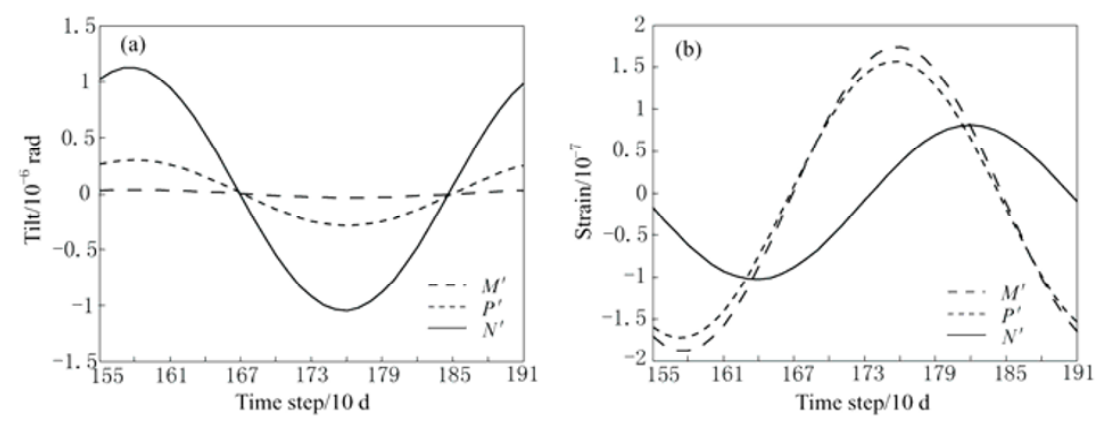

Figure 5 Tilt and strain of points $M^{\prime}, P^{\prime}, N^{\prime}$ vary with the time steps

tions, we will get different calculated annual variations, and to these two kinds of instruments (the baseline instrument and the pendulum instrument) installed at the same location, the calculation of annual variations are also different. This conclusion has been verified in actual observation. The annual variation of tilt and strain observed by the Taian seismic station are shown in Figure 6. The extensometer and water piper meter are types of the baseline instrument. The length of the baseline is $10.8 \mathrm{~m}$. The quartz pendulum is based on the principle of the pendulum instrument. As the actual structure of the tunnel in the Taian seismic station is very complicated the direction of tilt and strain has close relation to the location of instrument. So in this paper, we only consider the annual variation of tilt and strain and neglect the direction of them. Either in Figure 6a or in Figure $6 \mathrm{~b}$ the magnitude of annual variation of tilt is $10^{-6} \mathrm{rad}$. But there are some differences. The magnitude of annual variation of strain is $10^{-7}$ in Figure $6 \mathrm{c}$ and there are also some differences. These

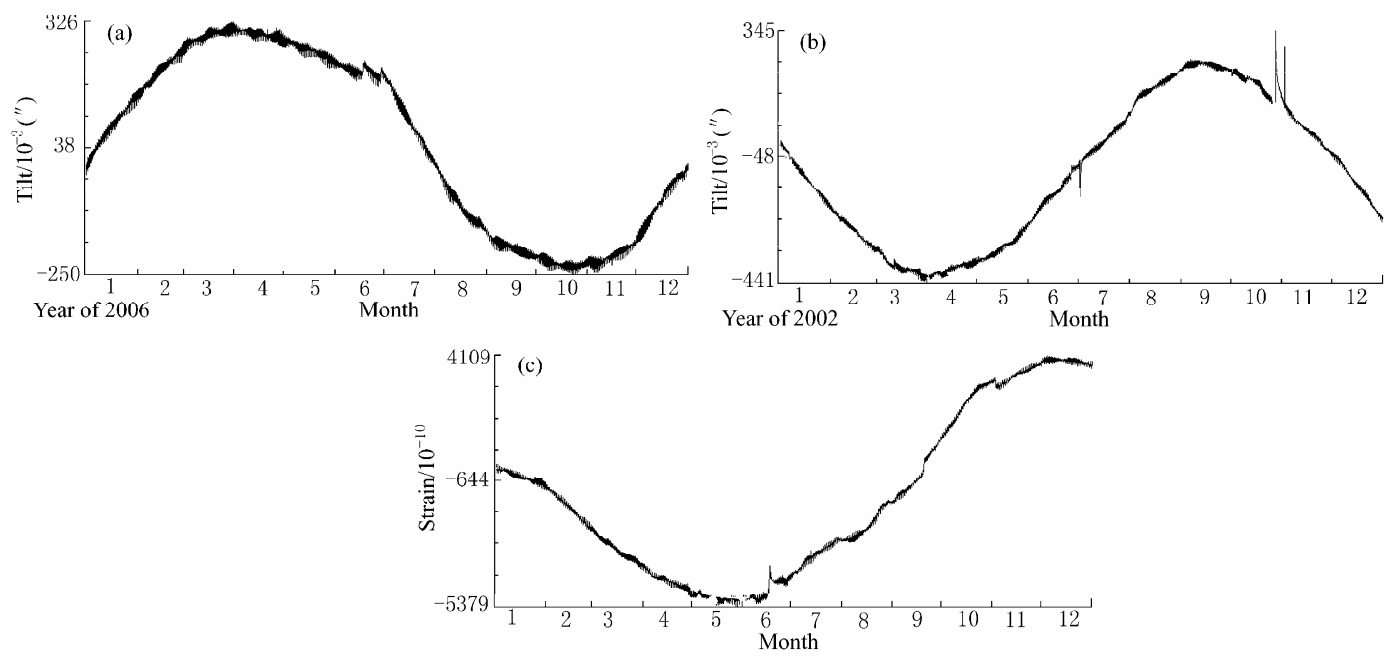

Figure 6 Observation results at Taian seismic station

(a) Observation results of tilt with water pipe; (b) Observation results of tilt with quartz pendulum; (c) Observation results of strain with extensometer

observations illustrate that different instrument installed in the same tunnel and under the same environment will get various observation results. However, we must indicate that the changes of tilt and strain in Figure 6 not only due to annual temperature variation inside the tunnel but also have relation to the temperature coefficient of instrument themselves. In actual seismic stations, 
because of the complicated structure of the tunnel and some other factors, different instruments will get various observation results and even the same instrument will have distinction observation results if installed at different location within the same tunnel. So selecting an appropriate location to install instrument is very important.

In this paper, we consider the medium as linear elastic and give some hypothesis when we establish the simple model for calculation. The results show that even a small perturbations of annual temperature inside the tunnel $\left(\sim 0.2^{\circ} \mathrm{C}\right)$ will produce a large changes of tilt and strain $\left(\sim 10^{-7}\right)$ because this variation can be observed actually. Although according to the requirement of construction standard of seismic station in China (2004), annual temperature variation amplitude smaller than $0.5^{\circ} \mathrm{C}$ is qualified, for better reduction of annual changes in deformation, the annual temperature variation inside the tunnel should be further reduced as far as possible and maintaining a constant temperature is the best. For either the baseline instrument or the pendulum instrument, the modeling can give guidelines in construction of the tunnel and installation of the instruments to reduce the effect of annual temperature variation inside the tunnel.

If the actual condition is more complicated and the medium is heterogeneity or there is topography on the surface, the magnitude and phase of temperature will be more complicated (CAO and SHI, 2005). However, with a variety of interference factor superposition, the fluctuations will be greater than the results of our calculation. This conclusion is also correct.

\section{Conclusions}

In this paper, finite element method (FEM) is employed to calculate the tilt and strain due to annual temperature variation inside the tunnel. The results show that even a small perturbations of temperature inside the tunnel $\left(\sim 0.2^{\circ} \mathrm{C}\right)$ will produce a large changes of tilt and strain $\left(\sim 10^{-7}\right)$. The distribution of thermal strain is uneven at different location. The changes of tilt and strain are larger at the corner or the end of the tunnel because heat convergence and diffusion is obvious there. The observation results of baseline instrument and pendulum instrument are distinctive. The changes of tilt and strain observed by the same instrument are various at different location.

The conclusion of our research is very useful to design and construction of tunnel, and installation of observation instruments. Though the construction standard of seismic station in China (2004) has regulated the magnitude of temperature inside the tunnel (the annual temperature variation is no more than $0.5^{\circ} \mathrm{C}$ ), it does not means that if meet the requirement we can eliminate the effect of annual temperature variation. To improve the quality of observation, seismic station needs to take further measures to reduce the annual temperature variation inside the tunnel. When the existence of small annual temperature variation is inevitable, we should consider the thermal deformation on the design of the tunnel and instrument installation, that is, the horizontal tunnel should be symmetrical to the shaft as far as possible and avoid setting baseline points at the corner or the end of the tunnel. For the baseline instrument, we can reduce the effect of annual temperature variation inside the tunnel if we set baseline points symmetrical to the shaft. On the other hand, for the pendulum instrument, the location of its installation is an important issue. We should select the location where the annual temperature variation is the smallest and avoid the corner or the end of the tunnel where heat convergence and diffusion are obvious.

\section{References}

CAO Jian-ling and SHI Yao-lin. 2005. Stress and tilt induced by annual variation of surface temperature [J]. Chinese Journal of the Graduate School of the Chinese Academy of Sciences. 22(3): 303-308 (in Chinese). 
China Earthquake Administration. 2004. Earthquake Industry Standard of People's Republic of China: Seismic Station Construction Norms Crustal Deformation Station (DB/T 8.1-2003) [S]. Beijing: Seismological Press: 1-11 (in Chinese).

Garavaglia M, Moro G D, Zadro M. 2000. Radon and tilt measurements in a seismic area: Temperature effects [J]. Physics and Chemistry of the Earth, 25(3): 233-237.

HU Wei-jian, ZHUANG Jun-shan, XIE Zhi, et al. 2002. Experimental result and theoretic analysis concerning the borehole strain responding to the load on Earth's surface [J]. Earthquake, 22(3): 95-104 (in Chinese).

LU Ming-wan and LUO Xue-fu. 1990. Foundation of Elastic Theory [M]. Beijing: Tsinghua University Press: $372-396$ (in Chinese).

Moro G D and Zadro M. 1998. Subsurface deformations induced by rainfall and atmospheric pressure: tilt/strain measurements in the NE-Italy seismic area [J]. Earth Plane Sci Lett, 164(1): 193-203.

MOU Ya-yuan. 1999. Processing of observation of P-C type strain meter at Xichang station and effect test [J]. Northwestern Seismological Journal, 21(3): 337-339 (in Chinese).

SHI Zhi-long and WU Shu-chao. 2007. Research of vertical pendulum tilt meter calibration method [J]. Science of Surveying and Mapping, 32(4): 63-64 (in Chinese).

SUN Yun-jun, LI Jie, CAO Jian-ling, et al. 2008. The simulation and comparative analysis for the strain and tilt of the seismic station of Taian in Shandong Province [G]//Institute of Earthquake Science of China Earthquake Administration, China Seismic Networks Center, The Seismological Society of China. Chinese Earthquake Prediction Research. Beijing: Earthquake publishing house: 460-466 (in Chinese).

Turcotte D L and Schubert G. 1986. Geodynamics: Second Edition [M]. Cambridge: Cambridge University Press: $125-168$.

WANG Mei and JI Ai-dong. 2000. Anomaly index study of fixed deformation measurement in Shandong Province [J]. Journal of Seismological ResearchI, 23(4): 398-404 (in Chinese).

WANG Mei, LI Feng, KONG Xiang-yang, et al. 2004a. Identification of disturbance of digital deformation observations [J]. Journal of Geodesy and Geodynamic, 24(1): 94-98 (in Chinese).

WANG Xiu-ying, LI Hai-liang, ZHOU Zhen-an. 2005. The realization of network of high precision, multi-component caved ground strain gauge [J]. Seismological and Geomagnetic Observation and Research, 26(5): 121-125 (in Chinese).

WANG Yue-feng, ZHANG Xiu-ping, LI Hai-xiao, et al. 2004b. Study on the extracting the information of the earthquake precursor abnormal about earth strain [J]. Seismological and Geomagnetic Observation and Research. 25(1): 35-40 (in Chinese).

XIAO Jun, ZHANG Jun, MO Yi-min, et al. 2005. High precision vertical pendulum tiltmeter based on spacial N-body central configuration [J]. China Mechanical Engineering, 16(11): 949-951 (in Chinese).

Zadro M, Plenizio E, Ebblin C, et al. 1988. Influence of groundwater table level variations and of rainfall on tilting in the Friuli area, N-E Italy [J]. Actu Geophysicu Polonicu, 35: 323-338.

Zeng R S. 1984. Introduction to Solid Geophysics [M]. Beijing: Science Press: 351-356 (in Chinese). 